\title{
Role of attention and translation in conflict resolution: implications for Stroop matching task interference
}

\author{
Walter Machado-Pinheiro ${ }^{1}$, Eliane Volchan ${ }^{2}, J^{\prime}$ aime Vila ${ }^{3}$, Elisa C. Dias ${ }^{4}$, Isabel Alfradique ${ }^{1}$, \\ Letícia de Oliveira $^{1}$, Mirtes G. Pereira ${ }^{1}$, and Isabel A. David ${ }^{1}$ \\ 1- Universidade Federal Fluminense, Niterói, RJ, Brazil \\ 2- Universidade Federal do Rio de Janeiro, Rio de Janeiro, RJ, Brazil \\ 3- Universidad de Granada, Granada, AN, Spain \\ 4- Nathan S. Kline Institute for Psychiatric Research, Orangeburg, NY, USA
}

\begin{abstract}
We studied the influence of attention on the timecourse of Stroop-like conflict. Thirty-two volunteers performed a Stroop matching task in which they had to compare either the color $(n=16)$ or meaning $(n=16)$ of two stimuli. The first stimulus was always a color-name printed in yellow, red, or blue (i.e., Stroop stimulus), and the second stimulus was either a color-bar (Experiment 1) or color-word in white ink (Experiment 2). Stimulus onset asynchrony (SOA) was varied parametrically. Interference by incongruent Stroop stimuli was clearly modulated by SOA manipulation in both cases. The results are discussed in terms of interactions between translational and attentional models in which the degree of Stroop-like interference is attributed to time implementation of attentional mechanisms during color-to-color and color-to-word matching contexts. Keywords: attention, Stroop task, timecourse, reaction time, cognitive processes, matching
\end{abstract}

Received 3 May 2010; received in revised form 19 October 2010; accepted 22 October 2010. Available on line 28 December 2010

\section{Introduction}

The complex environment in which we live makes distinguishing relevant from irrelevant information necessary. Selective attention limits neural processing to items that are currently relevant to behavior. To prevent crosstalk and interference, controlled attention must select relevant information, and irrelevant or distracting information must be suppressed. The Stroop task (Stroop, 1935 ) is widely used to investigate competition in cognitive processing (MacLeod, 1991). The Stroop effect relies on

Walter Machado-Pinheiro, Departamento Interdisciplinar de Rio das Ostras, PóloUniversitário de Rio das Ostras, Universidade Federal Fluminense, Brasil. Eliane Volchan, Programa de Neurociências, Instituto de Biofísica Carlos Chagas Filho, Universidade Federal do Rio de Janeiro, Brasil. Jaime Vila, Departamento de Personalidad, Evaluación Psicológica y Tratamiento, Facultad de Psicología, Universidad de Granada, España. Elisa C. Dias, Program in Cognitive Neurosciences and Schizophrenia, Nathan S. Kline Institute for Psychiatric Research, Orangeburg, NY, USA. Letícia de Oliveira, Mirtes G. Pereira, and Isabel A. David, Departamento de Fisiologia e Farmacologia, Instituto Biomédico, Universidade Federal Fluminense, Brasil. Correspondence regarding this article should be directed to: Walter Machado-Pinheiro, Departamento Interdisciplinar de Rio das Ostras, Pólo Universitário de Rio das Ostras / Universidade Federal Fluminense, Rua Recife, s/n, Jd. Bela Vista, Rio das Ostras - RJ, CEP 28.890-000; Tel/Fax: +55 (22) 2760 0848; E-mail: walter@vm.uff.br the observation that volunteers need more time to name the color ink of incongruent color-words (i.e., the word "blue" in red ink) than congruent color-words (i.e., the word "blue" in blue ink) or neutral ones (e.g., a sequence of colored "X"s). The increased reaction time (RT) of incongruent trials, referred to as Stroop interference, is considered a hallmark of the intrusion of task-irrelevant information in the context of selective attention. Many clinical and nonclinical studies have used Stroop-like tasks to assess frontal lobe function and the imposition of higher-order control to inhibit or suppress the processing of irrelevant stimuli, confirming the relationship between the Stroop task and the attentional executive system (Bruchmann, Herper, Konrad, Pantev, \& Huster, 2010; Krabbendam et al., 2009; Kravariti et al., 2009; Ungar, Nestor, Niznikiewicz, Wible, \& Kubicki, 2010).

The original Stroop task has countless variations. In the present study, we focused on the Stroop matching task, in which congruent and incongruent Stroop stimuli are presented with either a color-patch or another colorword in neutral ink. Volunteers compare different attributes of both stimuli and indicate whether they match or not. Although the interpretation of the results may be controversial, all Stroop matching task studies have confirmed the existence of Stroop-like interference (Dyer, 1973; Flowers, 1975; Goldfarb \& Henik, 2006; Luo, 1999; Treisman \& Fearnley, 1969). 
Interstimulus intervals (ISIs) or foreperiods play a central role in RT experiments and represent the periods of time when preparatory phases of reaction processes mainly occur (Niemi \& Nataanen, 1981). Volunteers use the foreperiod to prepare for stimulus occurrence and allocate controlled resources to optimize perceptual, cognitive, and motor performance (Fernandez-Duque \& Posner, 1997; Weber, Biscaldi, \& Fischer, 1995). In this sense, the use of variable intervals (Stimulus Onset Asynchrony, SOA) between stimuli in Stroop matching tasks represents an effective way of investigating the role and implementation of controlled processes involved in the suppression of distracter interference: long intervals would favor the implementation of such controlled processes. Nonetheless, timecourse studies dealing with Stroop-like interference are sparse, and most of the Stroop matching tasks have used simultaneous stimuli presentation (Dyer, 1973; Goldfarb \& Henik, 2006; Experiment 1 in Luo, 1999; Treisman \& Fearnley, 1969). Flowers (1975) was the first to introduce variable SOAs between the Stroop stimulus (used as the first stimulus, $\mathrm{S} 1)$ and the probe (S2, two color-patches) in a Stroop matching task. Subjects had to match the word of S1 to the color of S2 and press the corresponding key. Thus, different from the original Stroop study (1935), color (not word) was the distractive attribute of the Stroop stimulus. In his study, four different SOAs (ranging from 120 to $800 \mathrm{~ms}$ ) were used in a blocked array. The main result was interference of the $\mathrm{S} 1$ color (i.e., a reverse Stroop effect) only at short intervals.

The short list of studies that introduced time intervals in Stroop-like matching tasks includes those of Simon and colleagues (Simon \& Baker, 1995; Simon $\&$ Berbaum, 1988). However, in those studies, only an SOA of $2500 \mathrm{~ms}$ was used; therefore, the timecourse of interference could not be addressed. Other studies used variable SOAs. Aside from the fact that the Stroop attributes (color and word) had been segregated (i.e., not a bidimensional Stroop stimulus), a matching task was not required, and volunteers only had to respond orally (M.O.Glaser \& Glaser, 1982) or by pressing keys (Sugg $\&$ McDonald, 1994) in response to either a color-patch or color-word in white. Thus, whereas one attribute was used as a target, the other (distracter) attribute was presented before, concurrently with, or after the target only to disrupt performance. Chajut, Schupak, and Algom (2009) showed that the use of either bidimensional or spatially segregated Stroop attributes impose different task demands in attentional paradigms, thereby modulating the degree of interference (Chajut et al., 2009).

In addition to Flowers (1975), only Luo (1999; Experiment 2) introduced variable SOAs (from 1000 to $2500 \mathrm{~ms}$ ) in a Stroop matching task. In his "meaning decision task", subjects compared the ink of a bar to the meaning of a colored-word, regardless of its surface color, and pressed one of two keys corresponding to "same" or "different". In the "visual decision task", subjects compared the color ink of the two stimuli. When stimuli were presented simultaneously (Experiment 1), interference was found for the "same" response and only in the "meaning decision task," in which physical comparisons between stimuli were not possible, and a between-match (color-to-word) was required. In the "visual decision task," in which a color-to-color match was used, no significant interference was found. This pattern of results is consistent with the general concept of the translation model proposed by Virzi and Egeth (1985), in which interference occurs in tasks in which translation from one processing system to another is necessary. However, when SOAs were introduced in Luo's study (Experiment 2), interference gradually decreased in the "meaning decision task", and small but significant interference was found in the "visual decision task". He concluded that interference was attributable to conflict occurring at the semantic-level representation. Importantly, the SOAs adopted by Luo (1999) were long, and thus he could not study the gradual implementation of controlled mechanisms necessary to execute the tasks.

The aim of the present study was to compare the timecourse of Stroop matching tasks in color-to-color (visual) and color-to-word (meaning) decision contexts. Similar to Flowers (1975), we used the classic Stroop stimulus as S1. However, in our study, volunteers had to match the color of S1 (and not the word, as Flowers did) to a color-patch (Experiment 1) or to a word in white ink (Experiment 2). Thus, similar to the original Stroop task, the word was the irrelevant attribute. In both experiments, variable SOAs, including short SOAs, were introduced to investigate the gradual implementation of controlled processes to gate the distracter word. To our knowledge, this is the first study to test several aspects of Stroop matching in a single paradigm: $(i)$ a bidimensional Stroop stimulus (congruent or incongruent) was used as S1, and "word" was the source of interference; (ii) variable SOAs were introduced, allowing the gradual imposition of controlled processes during the intervals; and (iii) within- and between-match strategies were used, and thus interference could be compared when translation was not necessary and when it was obligatory. By comparing our data to previous studies that used different experimental protocols, we expect to evaluate which theoretical accounts are more consistent and survive the aforementioned changes, allowing us to fill knowledge gaps in the literature.

\section{Experiment 1}

In Experiment 1, the first stimulus (S1) was a colored-word (i.e., the Stroop stimulus), and the second stimulus (S2) was a color-bar. Subjects were told to compare the color of both stimuli and decide whether 
they had the same colored surface regardless of the word meaning (i.e., a within-mode comparison). In this case, conflict is present in S1. On incongruent trials, the word acts as a distracter and should interfere in the color-matching decision. Our prediction was that the duration of the interval would modulate the magnitude of Stroop interference: short intervals would favor sensorial (visual) match strategies, and long intervals would favor the allocation of a controlled mechanism to overcome the distracter attribute of S1.

\section{Methods}

\section{Participants}

Sixteen subjects (five male, 11 female), with a mean age of 21.8 years $(S D=3.5$ years), participated in the study. They were graduate students in biomedical/ biological sciences. All subjects were right-handed, determined by the Edinburg Inventory (Oldfield, 1971), and had normal or corrected-to-normal vision. They were naive with respect to the goals of the study. All procedures were approved by the local ethics committee, and written informed consent was obtained from participants prior to the study.

\section{Apparatus}

The experiment was conducted in a sound-attenuated room under dim ambient light. Subjects sat in front of a 14 inch video monitor $\left(0.2 \mathrm{~cd} / \mathrm{m}^{2}\right.$ luminance $)$, with their head positioned by means of a chin- and frontrest approximately $57 \mathrm{~cm}$ from the display. A computer running customized software (written with the MEL2 language; Psychology Software Tools Inc.) timed the presentation of the stimuli and recorded the key presses.

\section{Procedure}

Fig. 1 illustrates the temporal sequence of each trial. Each trial began with the presentation of an empty circle used as a fixation point (FP). Simultaneously, an auditory warning sound $(2000 \mathrm{~Hz}, 67 \mathrm{~dB}, 50 \mathrm{~ms})$ was emitted. After approximately $700 \mathrm{~ms}(700 \pm 100$ $\mathrm{ms}), \mathrm{S} 1$ was presented for either 100 or $300 \mathrm{~ms}, 1.5^{\circ}$ above the FP. S1 was a colored-word (YELLOW, RED, or BLUE), printed in yellow, red, or blue ink (i.e., the Stroop stimulus). Each letter measured $0.9^{\circ} \times 0.9^{\circ}$. The Stroop stimulus could be (i) congruent (the colorword and color ink were compatible; e.g., the word YELLOW in yellow ink) or (ii) incongruent (the two attributes were incompatible; e.g., the word YELLOW in red ink). After a variable time interval, S2 appeared in the same position where $\mathrm{S} 1$ had been previously presented $\left(1.5^{\circ}\right.$ above the FP). S2 was a bar $\left(0.9^{\circ} \times\right.$ $5.4^{\circ}$ ), printed in yellow, red, or blue ink and remained on the screen until the manual response occurred. The interval between S1 offset and S2 onset (ISI) could be either 100 or $300 \mathrm{~ms}$. The S1 duration and ISI occurred randomly and with the same probability throughout the experiment. Therefore, the combination of S1 duration and ISI produced three different SOAs: 200, 400, and $600 \mathrm{~ms}$ (SOA-200, SOA-400, and SOA-600, respectively). As mentioned above, Luo (1999) showed that for simultaneous stimuli presentation under similar conditions, color-to-color comparisons did not produce any interference, and other slightly different studies also reached the same conclusion (e.g., Treisman \& Fearnley, 1969; M.O.Glaser \& Glaser, 1982; Sugg \& McDonald, 1994; Durgin, 2000). Therefore, we decided to study the timecourse of color-to-color matches for SOAs longer than $0 \mathrm{~ms}$ to complement those findings. Moreover, notice that the SOA- 400 could be achieved either by an S1 duration of $100 \mathrm{~ms}$ and ISI of $300 \mathrm{~ms}$ or by an S1 duration of $300 \mathrm{~ms}$ and ISI of $100 \mathrm{~ms}$. This differential presentation of the SOA-400 was used to verify the influence of $\mathrm{S} 1$ duration on the degree of interference.

Subjects were instructed to ignore the meaning of the colored-word and compare the colored surfaces of both stimuli. If the two stimuli had the same colored surface, then they should press a key with the right index finger as soon as $\mathrm{S} 2$ was detected (manual reaction time- MRT). If colors were different, then no response was required. This was the first Stroop matching task study in which subjects were required to press only one key to generate RTs (a go/no-go procedure). Previous studies used at least two different response options (e.g., "same" and "different") - a choice RT procedure (Dyer, 1973; Goldfarb \& Henik, 2006; Luo, 1999). A careful inspection of those papers revealed that reliable and consistent Stroop interference (i.e., longer RT for incongruent trials) was always obtained for the "same" response. The results were less consistent when a "different" response was required (compare the Dyer

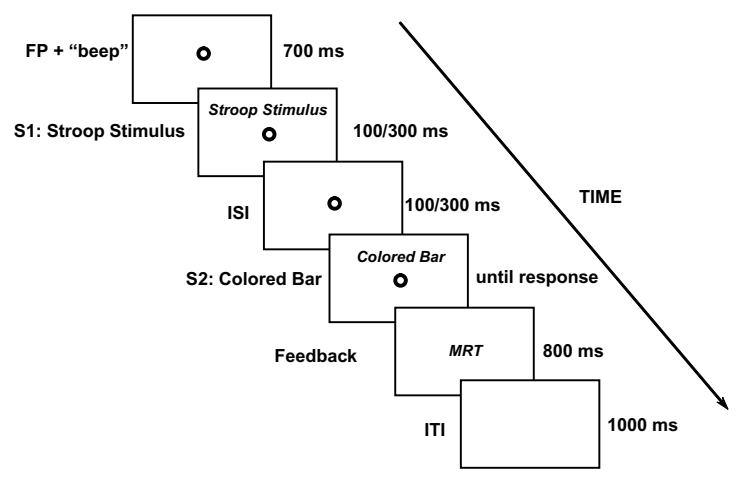

Figure 1. Schematic representation of the event sequence in each trial of Experiment 1. The first stimulus (S1; the Stroop stimulus) was a colored-word (YELLOW, RED, or BLUE) also in yellow, red, or blue ink. In the incongruent condition, the word and the color were not compatible (e.g., the word YELLOW in blue ink). The second stimulus (S2) was a color-bar. Subjects had to compare the color of S1 with the color of S2. S1 and S2 were separated by different Stimulus Onset Asynchronies (SOAs). MRT, manual reaction time; ITI, intertrial interval. 
[1973 word-color paradigm, Dyer [1973] color-word paradigm, Goldfarb \& Henik [2006], and Luo [1999]). To disambiguate the results obtained with "same" vs. "different" choices, we emphasized and increased the number of "same" responses, in which a robust effect has always been obtained. Nonetheless, despite the absence of an overt response, the "different" condition occurred in $25 \%$ of our trials, and it was important to minimize anticipatory behavior by the subjects. Therefore, go-trials corresponded to $75 \%$, and no-go trials corresponded to $25 \%$.

Each session lasted approximately $20 \mathrm{~min}$ and consisted of four blocks of 48 trials each, with 1 or 2 min of rest between blocks. Each block contained the same number of congruent and incongruent trials in a randomized order. Anticipatory responses (MRT $<100 \mathrm{~ms}$ ), slow responses (MRT $>1500 \mathrm{~ms}$ ), and incorrect key-press responses (key press in a no-go trial) were considered errors and were excluded from further analyses. Visual feedback (response latency and errors) was presented on the screen for $800 \mathrm{~ms}$. The subsequent trial began after an additional intertrial interval (ITI) of $1000 \mathrm{~ms}$.

MRTs were analyzed by a Congruency (congruent vs. incongruent $) \times \mathrm{S} 1$ Duration $(100$ vs. $300 \mathrm{~ms}) \times$ ISI (100 vs. $300 \mathrm{~ms})$ repeated-measures analysis of variance (ANOVA), with median RT of correct trials as the dependent variable. When appropriate, post hoc analysis was performed using the Newman-Keuls test. The alpha level of significance was .05 .

\section{Results}

The overall mean percentage of errors was $2.8 \%$ (only 86 errors in 3,072 trials). Omission errors in gotrials $(n=24)$ and commission errors in no-go trials ( $n=62)$ tended to be equally distributed among the conditions. Such error patterns do not support a speedaccuracy trade-off interpretation of our data. Moreover, because the error rate was very low, we did not conduct any ANOVA to analyze them.

The ANOVA of the MRT data revealed main effects of Congruency $\left(F_{1,15}=52.19, p<.001\right)$ and ISI $\left(F_{1,15}=72.38\right.$, $p<.001)$. The S1 duration $\times$ ISI interaction $\left(F_{1.15}=25.38\right.$, $p<.001)$ and three-way interaction $\left(F_{1,15}=4.79, p=.045\right)$ were also significant. MRTs were shorter for congruent than incongruent trials (304 vs. $320 \mathrm{~ms}$, respectively). The ISI factor showed that MRTs were shorter for the ISI of $300 \mathrm{~ms}$. The three-way interaction revealed that the difference between incongruent and congruent trials (i.e., interference) was modulated by S1 duration and ISI. Fig. 2 plots the average MRT obtained for incongruent and congruent trials for all combinations of S1 durations and ISIs. The post hoc analysis showed that interference was significant in both cases of SOA-400: $27.1 \mathrm{~ms}(p<.001)$ for S1 duration of $100 \mathrm{~ms}$ and ISI of $300 \mathrm{~ms}$, and 16.3

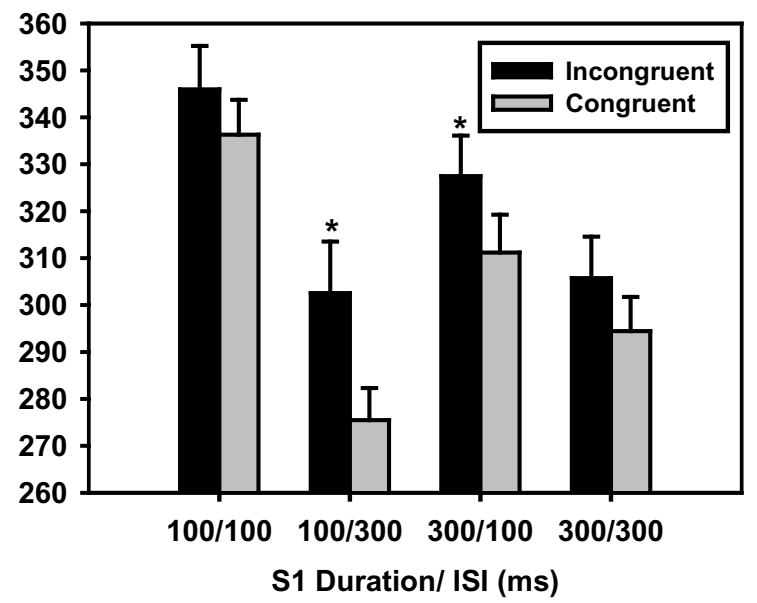

Figure 2. Average median reaction times for the different combinations of S1 duration and ISI. The error bars indicate the standard error of the mean. Asterisks indicate statistically significant differences between congruent and incongruent trials $\left({ }^{*} p<0.05\right)$.

ms $(p<.001)$ for S1 duration of $300 \mathrm{~ms}$ and ISI of 100 $\mathrm{ms}$. A trend was found toward significant interference at SOA-200 (9.7 ms, $p=.078)$, with no significant effect at SOA-600 $(11.3 \mathrm{~ms}, p=.101)$.

A secondary issue of this experiment was also addressed, namely the interference modulated by S1 duration. We compared the degree of interference using both combinations of S1 duration/ISI used for SOA400. A paired $t$-test revealed that the values did not differ $(p=.164)$. Altogether, these data indicate that in a Stroop color-matching task, SOA (the effective time between the two stimuli), more than the S1 duration or ISI separately, is able to exert an important influence in modulating distractive interference.

\section{Discussion}

The MRT data confirmed that Stroop-like interference in a color-matching task is influenced by temporal manipulations. Our results have implications for theoretical models of Stroop-like interference. The degree of interference was small and was found only at SOA-400. Notably, in this experiment, a color-to-color match was required (i.e., a within-mode comparison). Translational accounts propose that Stroop-like interference occurs only when translation between attributes is necessary for response execution. Consequently, our data cannot be completely explained by those accounts. Small but significant interference of words in color-to-color comparisons has been found by others. By means of a Stroop pointing matching task, Durgin (2000) found that interference occurred in his color identification condition, despite the fact that translation was not necessary. However, interference increased significantly when word-to-color comparisons were 
required. These and other results from the same author (Durgin, 2003) were used as evidence to support translational models, despite the existence of small but significant interference obtained from within-mode comparisons.

As mentioned above, in his color-to-color matching task (i.e., match a patch to the ink of a colored-word), Luo (1999) found that interference was absent with simultaneous stimuli presentation. He proposed that at short intervals, matching could be based on the physical aspects of stimuli (colors) before any interference of verbal codes. This is similar to the results found at SOA-200 in our study. According to Luo, physical-matching strategies were no longer possible for longer intervals, causing semantic competition to occur, similar to our results with SOA-400. In fact, evidence indicates reduced efficiency in a physical-matching strategy as the ISI increases, reflecting the decay of visual information $(\mathrm{Lu}$, Neuse, Madigan, \& Dosher, 2005; Posner, Boies, Eichelman, \& Taylor, 1969). For example, Posner et al. (1969) tested physical and name identity matches between pairs of letters (e.g., Aa or AA) with different intervals between them $(0,500,1500$, and $2000 \mathrm{~ms})$. They found robust facilitation for the physical match (AA) compared with the semantic match (Aa) with simultaneous presentation and a decay of this facilitation over time. M.O.Glaser and Glaser (1982) also obtained timecourse results that support such an interpretation. In Experiment 3 of their study, they showed that naming the color of a patch was facilitated when it was preceded $200 \mathrm{~ms}$ or earlier by another irrelevant, but congruent patch. When the interval increased, pictorial information lost efficiency, and no facilitation was found. Such a timecourse is similar to that found in Experiment 1 of the present study and supports the idea that matches based on the physical aspects of stimuli (colors) are favored at short intervals. Finally, the absence of interference observed at SOA-600 indicates that, by that time, controlled attentional processes are efficient in gating the influence of an S1 distracter.

In summary, evidence from different sources support the notion that translational accounts alone are not able to explain the diversity of results observed in color-to-color matching when distinct intervals between stimuli are employed. Interference varies according to the task demands imposed by the intervals, which indicates the involvement of other mechanisms. Experiment 2 investigated how intervals influence Stroop-like interference in a color-to-word matching task in which translation is necessary.

\section{Experiment 2}

Experiment 1 revealed that the interference produced by the distracter word in a Stroop color-matching task was small and significant only at SOA-400. Because the only task requirement was color matching, subjects may have been able to direct attentional resources specifically to colors, without any obligatory translation. In Experiment 2, instead of a color-bar, a color-name written in white was used as S2 (a color-to-word match). Subjects had to transform the color of the Stroop stimulus into a verbal code (translation) to compare it with the word of S2. Our prediction was that the obligatory activation of competing verbal units to both S1 attributes (color and word) would increase the distracter interference mainly at short SOAs, and thus a different timecourse of interference was expected.

\section{Methods}

\section{Participants}

Sixteen subjects (six males, 10 females), with a mean age of 20.7 years ( $S D=1.7$ years), participated in the study. All other subject characteristics were identical to Experiment 1.

\section{Apparatus}

The apparatus used in Experiment 2 was exactly the same as Experiment 1.

\section{Procedure}

The experimental design was similar to Experiment 1. In Experiment 2, S1 was a Stroop stimulus (coloredwords printed in yellow, red, or blue), but S2 was a color-word (YELLOW, RED, or BLUE) printed in white that remained on the screen until the manual response was emitted. S1 was presented $1.5^{\circ}$ above the FP for $200 \mathrm{~ms}$, and $\mathrm{S} 2$ was presented $1.5^{\circ}$ below the FP. The intervals between $\mathrm{S} 1$ offset and $\mathrm{S} 2$ onset were 200 or $800 \mathrm{~ms}$, resulting in SOAs of 400 and 1000 ms. S1 and S2 could also be presented simultaneously (SOA-0), in which case both remained on the screen until the response was emitted by the subject. Volunteers were told to compare the color ink of S1 to the word of S2 and to press a key if they had the same meaning. The proportion of "go" and "no-go" trials was maintained at $75 \%$ and $25 \%$, respectively. In this study, SOA-400 was again used because it was the source of interference in the previous experiment. The longest SOA was increased to $1000 \mathrm{~ms}$ to allow deployment of controlled mechanisms. Finally, SOA-0 was introduced to serve as baseline. Because S1 and S2 were presented simultaneously, subjects had no time before $\mathrm{S} 2$ onset to impose controlled attentional mechanisms to the distracter attribute of S1, and so maximal interference should occur.

MRTs were analyzed by performing a Congruency (congruent $v s$. incongruent) $\times \operatorname{SOA}(0,400$, and $1000 \mathrm{~ms}$ ) repeated-measures ANOVA, with median RT of correct trials as the dependent variable. When appropriate, a post hoc analysis was performed using the Newman-Keuls test. The alpha level for statistical significance was .05 . 


\section{Results}

Similar to Experiment 1, errors were rare and not sufficient to proceed with an Error-ANOVA: 150 errors in 3,456 trials $(4.3 \%)$. Omission errors in go-trials $(n=$ 41) were more frequent for the incongruent condition (32 vs. 9), but commission errors tended to be equally distributed among the conditions. Such a pattern of results does not support a speed-accuracy trade-off interpretation of our data.

The ANOVA of MRT data showed that Congruency and SOA were significant sources of variance $\left(F_{1,15}=\right.$ $55.42, p<.001$, and $F_{2,30}=299.34, p<.001$, respectively). The Congruency $\times$ SOA interaction was also significant $\left(F_{2,30}=70.89, p<.001\right)$. MRTs obtained for congruent trials were shorter than those obtained for incongruent trials (464 vs. $564 \mathrm{~ms}$ ). Post hoc analysis showed that MRTs tended to decrease as SOA increased (734 vs. 443 vs. $365 \mathrm{~ms}$, respectively; $p<.001$ for all comparisons). Fig. 3 plots the average MRT for congruent and incongruent conditions at the different SOAs. Post hoc analysis revealed that MRTs for incongruent and congruent conditions were significantly different at SOA-0 (866 vs. $602 \mathrm{~ms}$, respectively; $p<.001$ ) and SOA-400 (480vs. $406 \mathrm{~ms}$, respectively; $p<.001$ ). At SOA-1000, the difference was inverted and was almost cionificant $(247$ ve $382 \mathrm{mc}$ recnertivaly $n=052)$

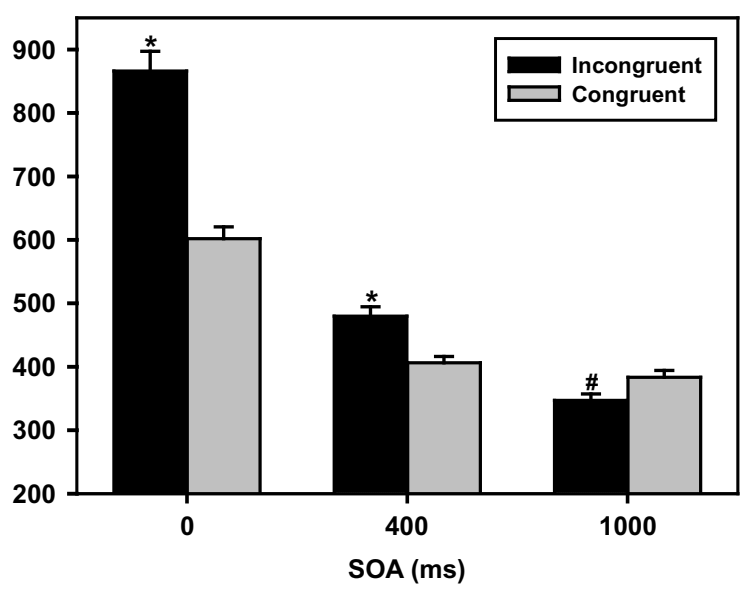

Figure 3. Average median reaction times for different SOAs in Experiment 2. The error bars indicate the standard error of the mean. Asterisks indicate statistically significant differences between congruent and incongruent trials $\left({ }^{*} p<.05\right)$. The hash sign refers to the marginal effect $(\# p=.053)$ found at $1000 \mathrm{~ms}$.

An additional paired $t$-test also revealed a significant difference in the Stroop effect magnitude between SOA-0 and SOA-400 (264 vs. $74 \mathrm{~ms}, p<.001)$.

\section{Discussion}

Our data confirmed that translation represents a critical step in Stroop interference. The amount of interference was $264 \mathrm{~ms}$ at SOA-0, $74 \mathrm{~ms}$ at SOA-400, and nonexistent at SOA-1000, which was significantly higher compared with Experiment 1. Moreover, the gradual decrease in interference with increasing SOAs indicates the timecourse of controlled mechanisms necessary to gate the distracter attribute of the Stroop stimulus.

Using different approaches, previous studies have found strong word interference in color-naming tasks when a color-word was presented temporally closer to a color-target patch (Appelbaum, Meyerhoff, \& Woldorff, 2009; M.O.Glaser \& Glaser, 1982). M.O.Glaser and Glaser (1982) found that $500 \mathrm{~ms}$ was not long enough to cancel the word influence, which contrasts with the results obtained at SOA-1000 in the present study. Their results were explained in terms of a failure to gate the intrusion of an irrelevant verbal code into central analyzers, disrupting the main task. The electrophysiological correlate of such behavioral data was recently presented in an event-related potential (ERP) study (Appelbaum et al., 2009). Similar timecourse results were obtained by Dyer (1971) in his color-naming task, in which volunteers had to name the ink color of a Stroop stimulus that was preceded (from 0 to $500 \mathrm{~ms}$ ) by a color-name in black (i.e., prime). He found that even after an interval of $500 \mathrm{~ms}$, incongruent primes were able to interfere with the color naming task. Therefore, convergent results support the hypothesis that voluntary attentional mechanisms can overcome the distracter information of a Stroop stimulus if enough time is allowed (i.e., $>500$ $\mathrm{ms}$ and $<1000 \mathrm{~ms}$ is the time necessary to suppress such verbal-distractive interference).

At $1000 \mathrm{~ms}$, not only was the Stroop effect abolished, but a reverse pattern was also obtained (i.e., faster responses for incongruent trials). A possible explanation for this result could be based on the negative priming phenomenon. Negative priming is defined as the slowing RT to the probe when it is somehow related to the preceding distracter dimension (Fox, 1995; Neill, 1977). In Experiment 2, subjects had to ignore the word attribute of $\mathrm{S} 1$ to compare the color of S1 with the meaning of S2. Notice that for congruent-go trials, the distracter dimension of S1 (the word to be ignored; e.g., the word BLUE in blue ink) had the same meaning as S2 (the word BLUE in white). However, for incongruentgo trials, the word presented as S1 (e.g., RED in blue) never had the same meaning as S2 (the word BLUE in white). As a result, negative priming could only occur in the congruent-go condition. Interestingly, negative priming was only observed at the longest SOA. In fact, negative priming is thought to be generated by controlled processes that gate the distracter information of S1, and time is necessary to achieve this (Houghton \& Tipper, 1994; Neill \& Westberry, 1987; Ortells, Abad, Noguera, \& Lupianez, 2001; Ortells, Fox, Noguera, \& Abad, 2003). Also important is that a negative prime could not be found in Experiment 1 because S2 was not a word, but rather a color-bar. Thus, the reverse 
pattern of results obtained at SOA-1000 strongly suggests that at this SOA the word attribute is gated by attention, inhibiting its access into central analyzers. In a recent paper, Hanslmayr et al. (2008) showed that RTs obtained in a "negative priming" condition (i.e., when the relevant dimension of a trial corresponded to the irrelevant attribute of the preceding trial; trial $n-1)$ were longer than those obtained for the other incongruent conditions, in which no relationship between previous and actual attributes were found. Their findings validate our interpretation and confirm that trials in which negative priming relationships occur are, in fact, under particular influences (Hanslmayr et al., 2008).

\section{General Discussion}

\section{General aspects}

The results obtained in Experiment 1 revealed the timecourse of interference produced by a distracter word during a color-matching task. Interference was not significant at SOA-200, was significant (although small) at SOA-400, and disappeared at SOA-600. In Experiment 2 , in which translation was needed, interference was higher, maximal for simultaneous presentation, and reduced significantly with increased SOAs.

A complete discussion of different models for Stroop-like interference was not the goal of this paper. However, our data allow some theoretical considerations. The results of Experiment 1 revealed reduced but significant interference, similar to Durgin (2000) in his "color ID - fixed location" condition (see Fig. 2 in Durgin, 2000). In both cases, color matches were required. Therefore, $(i)$ verbal processing of the Stroop stimulus must be gated by the attentional system, or (ii) no translation is necessary (a within-attribute comparison). In Experiment 2, a comparison was made between the color of the Stroop stimulus and the word used as S2, which significantly increased interference. In that case, color had to be translated into a word to allow the match, and therefore the verbal analyzer could not be gated. It increased the impact of the verbal information of $\mathrm{S} 1$, and the manual response was delayed. In the present study, S1 and the response were identical in both experiments. The only difference was the type of stimulus used as S2, and the comparison required a color-to-color match in Experiment 1 and color-to-word match in Experiment 2. To our knowledge, our study used the simplest manual response possible in Stroop matching tasks. Durgin (2003) also used a single keypress response to study the reverse Stroop effect, but his experimental design, procedures, and goals were entirely different from the present study. Despite the use of a go/no-go approach in our study, consistent and reliable interference was found, and, more importantly, it varied significantly in both experiments. Our data validate the use of go/no-go procedures in Stroop matching tasks. An advantage of such a procedure is a reduction in the number of conditions to be analyzed. It is a useful strategy that can be applied in studies in which a large number of conditions represent a strong limitation for data processing, such as ERPs or functional magnetic resonance studies that investigate Stroop-like interference (Mitchell, 2006; Zysset, Muller, Lohmann \&, von Cramon, 2001).

\section{Stroop matching task interference: role of translation}

Stroop matching tasks have contributed to many theoretical aspects related to Stroop-like interference. Treisman and Fearnley (1969) used a card version of the Stroop matching task to verify the role of selective attention and tested the hypothesis that ignoring a word is harder than ignoring a color. The study had four experimental conditions, including within- and between-attribute comparisons. Subjects should match the color or word of the Stroop stimulus with a colorword in black or with a sequence of colored "X"s. They found that performing the task with betweenattributes was more difficult than within the same mode. Thus, responses were delayed even when the word of the Stroop stimulus was matched with the sequence of colored "X"s (i.e., when color was the distractive attribute). This result indicates that the simplistic Stroop effect explanation involving attentional failure in gating the "word" in contrast to the "color" information needed further elaboration. These pioneering findings have been used by others to support the translational account of Stroop interference (W.R.Glaser \& Glaser, 1989; Virzi \& Egeth, 1985). In short, translation models hold that interference occurs when a response requires translation between the internal code of the relevant attribute to another code according to the response. Therefore, classic translational accounts predict that when two items belong to the same modality or share the same central analyzer, no translation is required and no interference is found.

Contrary to the original translational account and using manual responses, some authors showed that obtaining interference is possible even for color-tocolor comparisons, in which no translation is necessary (Durgin, 2000; Sugg \& McDonald, 1994). As previously mentioned, small but significant interference was observed by Durgin (2000) in the "color identification" condition of his Stroop pointing task, in which no translation was necessary. Moreover, Sugg and McDonald (1994) showed that when volunteers had to respond to the color by pressing a colored button on a touchscreen (no translation), interference was observed when an irrelevant word preceded the colored target by at least $200 \mathrm{~ms}$. The word did not interfere when it was presented simultaneously or after the colored target. However, the degree of interference obtained in these previous studies was significantly smaller than when 
translation was necessary (for details, see M.O.Glaser \& Glaser, 1982, and the "translated word-response task" of Sugg \& McDonald, 1994). These results fit well with our findings that the Stroop effect in color-to-color comparisons was clearly smaller than in color-to-word comparisons. This small color-to-color interference was also used to support the participation of mechanisms other than translation in Stroop-like conflict, such as response competition (Blais \& Besner, 2007; Durgin, 2000; Goldfarb \& Henik, 2006). Therefore, we cannot discard the possibility that response conflict between the distracter and the relevant dimensions may also contribute to the interference found in the present work. The behavioral approach used in the present and previous studies is limited in its ability to appropriately assess the contribution of conflicts at the stimuli representation or motor response level in Stroop matching tasks. In this sense, the use of ERPs has been successfully employed for this purpose (e.g., Szucs, Soltesz, \& White, 2009). Future ERP studies addressing this issue are necessary to separate brain correlates of stimulus and response conflict in Stroop matching tasks. We also demonstrated distinct timecourses for between- and within-matching tasks. For short SOAs, interference was maximal in the first case and not significant in the second. These results can be partially explained by translation models, although they do not provide a satisfactory explanation for the variety of findings obtained when intervals were inserted.

\section{Stroop matching task interference: role of attention}

The involvement of selective attention in Strooplike interference, directly or indirectly, had already been suggested by others, including those supporting other models. For example, Glaser and Glaser (1982) showed in their Experiment 1 that an irrelevant color patch did not interfere in a color reading task. However, in their Experiment 2, when the patch was used as a cue indicating the name to be read with $80 \%$ probability, interference by incongruent patches was found when they preceded the word by $\geq 200 \mathrm{~ms}$. Thus, the modulatory role of attention was clear. When more attention was devoted to the patch because of its high predictability, reading interference occurred. Additionally, Sugg and McDonald (1994) suggested the possible involvement of attention to explain the difference in the amount of interference obtained in similar conditions in two experiments. A remarkable reduction in interference was found from Experiment 1 to Experiment 2 when another incongruent condition (i.e., the so-called "incongruent never a response" condition) was introduced, increasing the incongruent-like conditions from $33 \%$ to $50 \%$ in Experiment 2. According to these authors, because incongruent trials were more frequent in Experiment 2 (in an attempt to attenuate distracting interference), "subjects may have attended less to the irrelevant stimulus in Experiment 2 than in Experiment 1, thereby decreasing the magnitude of inhibition" (Sugg \& McDonald, 1994, p. 668a, second paragraph).

Simon and Berbaum (1988) introduced a fixed interval between stimuli in a Stroop matching task. Subjects viewed the Stroop stimulus for 2,000 ms and $500 \mathrm{~ms}$ after the probe (i.e., a color-word in white or a colored patch) was presented. Subjects had to match the relevant attribute of the Stroop stimulus with the probe. Depending on the condition, the relevant attribute could be the word or the ink color. In a more detailed study, Simon and Baker (1995) extended the experiments of Simon and Berbaum (1988) by varying the order of stimuli presentation. Therefore, two different trials were produced: Stroop-first and probe-first. In both trials, depending on the instructions from the researcher, a within- or between-mode comparison was required. In short, previous studies revealed that interference was especially apparent in between-mode matches, favoring translational accounts. However, more detailed inspection reveals an interesting finding, namely that no interference was found in the Stroop-first trials of Experiments 1 and 2 in Simon and Baker(1995), whereas the same trials produced significant interference in the study by Simon and Berbaum (1988). An important procedural difference between these two studies was the experimental context. Within- and between-matches were blocked in the Simon and Baker (1995) study but not in the Simon and Berbaum (1988) study. In a blocked context, subjects knew beforehand which attribute of the Stroop stimulus should be attended or unattended and also the match that would be required (within or between) in each trial. Such predictability is impossible in a random within/between context. Moreover, because SOA was long, the attentional set could gate the distractive influence and abolish interference in the blocked context. In fact, to explain such discrepancies, Simon and Baker (1995) wrote, "Subjects could then focus on that attribute [the relevant one] and match it to the probe. There was no necessity to store the irrelevant attribute or even to attend to it" (Simon \& Baker, 1995, p. 1040b, second paragraph), highlighting the modulatory role of attentional mechanisms in Stroop-like interference. The influence of the blocked vs. non-blocked context in the imposition of attentional control was demonstrated by Machado-Pinheiro et al. (2004). Interference by a visual onset disappeared when a blocked context was used (Machado-Pinheiro, Faria, Gawryszewski, \& Ribeiro-do-Valle, 2004).

Virzi and Egeth (1985) showed that translation of an irrelevant code into the criterial dimension is not obligatory and occurs only when it is required by task demands. Translation and its distractive influence can be gated by task demands. They did not explicitly mention the word "attention" in their explanation, but we believe that such "gating" is executed by higher-order attentional processes. Other recent studies have also supported the 
major role of attention in the modulation of the Stroop effect (Bruchmann et al., 2010; Choi, Cho, \& Proctor, 2009). These studies concluded that the degree of attention/consciousness devoted to the Stroop attributes is important for modulating the impact of the distractive dimension on behavioral performance (interference). Our study extended this conclusion by showing that, once the distractive attribute was read and processed, approximately 600 to $1000 \mathrm{~ms}$ is necessary to reduce/ suppress its impact on RT performance.

Altogether, our results suggest that when colorto-word translation was not necessary (Experiment 1), attentional mechanisms could gate the verbal encoding and reduce the distracter verbal interference. However, when the color attribute had to be translated into verbal information, the verbal analyzer could not be easily blocked, and the distracter verbal information of S1 produced strong interference. Our data support models that postulate that interference is linked to conflicts in the internal representations of Stroop attributes, especially when translation is necessary. When this occurs, gating distracter inputs by the attentional control system and maintaining the overall task goal according to the instructions are more difficult. In fact, we believe that translation and attentional accounts are correct, and selective attention is critical for understanding how different analyzers can or cannot be blocked, depending on the experimental procedures and the matches required. Our interpretation is consistent with Roelofs (2003), who focused his computational model on the mechanisms underlying attentional control on word production and perception. He described the complex lexical processes involved before word articulation, such as lemma retrieval and word-form encoding, and considered that these processes are guided by controlled attentional mechanisms according to task goals (Roelofs, 2003). In a more recent paper, Roelofs (2010) reinforced his original account in which distractive words are only processed for a limited period of time before they are actively blocked. Our study indicates that this period of time would be between 600 to1000 ms.

\section{Concluding remarks}

Our data showed that regardless of whether translation is necessary and independent of the match required by the task, after a time interval, interference was no longer significant. Attentional mechanisms use this extra time to eliminate the distracter influence and solve the conflict. The results also indicate that such "gating" occurs gradually and that for long SOAs, inhibitory processes are able to block the entrance of the distractive word attribute into central analyzers. The blockade of the distracter dimensions may promote the negative priming effect observed when the target-word (in S2) corresponded to the distracter word (inhibited in S1) at SOA-1000, confirming the inhibitory nature of the controlled attentional processes observed in the present study. Consistent with such an interpretation, Stroop-like interference could be related to the longer time necessary to achieve full efficiency of higherorder processes necessary for countering the distractive influence. In conclusion, our results highlight the relevance of both attentional and translational accounts of interference and the timecourse observed in Stroop matching tasks. The results also indicate that attention controls and modulates conflict, particularly during color-to-word matches.

\section{Acknowledgments}

This research was supported by grants from CAPES, CAPES-DGU/MECD no 086/05, PRONEX-FAPERJ, and PROPP-UFF. We would like to thank an anonymous reviewer for his valuable comments and suggestions on an earlier version of the manuscript.

\section{References}

Appelbaum, L.G., Meyerhoff, K.L., \& Woldorff, M.G. (2009). Priming and backward influences in the human brain: processing interactions during the Stroop interference effect. Cerebral Cortex, 19, 2508-2521.

Blais, C., \& Besner, D. (2007). A reverse Stroop effect without translation or reading difficulty. Psychonomic Bulletin and Review, 14, 466-469

Bruchmann, M., Herper, K., Konrad, C., Pantev, C., \& Huster, R.J. (2010). Individualized EEG source reconstruction of Stroop interference with masked color words. Neuroimage, 49, 1800-1809.

Chajut, E., Schupak, A., \& Algom, D. (2009). Are spatial and dimensional attention separate? Evidence from Posner, Stroop, and Eriksen tasks. Memory and Cognition, 37, 924-934.

Choi, J.M., Cho, Y.S., \& Proctor, R.W. (2009). Impaired color word processing at an unattended location: evidence from a Stroop task combined with inhibition of return. Memory and Cognition, 37, 935-944.

Durgin, F.H. (2000). The reverse Stroop effect. Psychonomic Bulletin and Review, 7, 121-125.

Durgin, F.H. (2003). Translation and competition among internal representations in a reverse Stroop effect. Perception and Psychophysics, 65, 367-378.

Dyer, F.N. (1971). The duration of word meaning responses: Stroop interference for different preexposures of the word. Psychonomic Science, 25, 229-231.

Dyer, F.N. (1973). Same and different judgments for word-color pairs with "irrelevant" words or colors: evidence for word-code comparisons. Journal of Experimental Psychology, 98, 102-108.

Fernandez-Duque, D., \& Posner, M.I. (1997). Relating the mechanisms of orienting and alerting. Neuropsychologia, 35, 477-486.

Flowers, J.H. (1975). "Sensory" interference in a word-color matching task. Perception and Psychophysics, 18, 37-43.

Fox, E. (1995). Negative priming from ignored distractors in visual selection: a review. Psychonomic Bulletin and Review, 2, 145-173.

Glaser, M.O., \& Glaser, W.R. (1982). Time course analysis of the Stroop phenomenon. Journal of Experimental Psychology, 8, 875-895.

Glaser, W.R., \& Glaser, M.O. (1989). Context effects in Strooplike word and picture processing. Journal of Experimental Psychology: General, 118, 13-42.

Goldfarb, L., \& Henik, A. (2006). New data analysis of the Stroop matching task calls for a reevaluation of theory. Psychological Science, 17, 96-100.

Hanslmayr, S., Pastotter, B., Bauml, K.H., Gruber, S., Wimber, M., \& Klimesch, W. (2008). The electrophysiological dynamics of interference during the Stroop task. Journal of Cognitive Neuroscience, 20, 215-225.

Houghton, G., \& Tipper, S.P. (1994). A model of inhibitory 
mechanisms in selective attention. In D. Dargenbach \& T.H. Carr (Eds.), Inhibitory mechanism in attention, memory and language (pp. 53-112). San Diego: Academic Press.

Krabbendam, L., O’Daly, O., Morley, L.A., Van Os, J., Murray, R.M., \& Shergill, S.S. (2009). Using the Stroop task to investigate the neural correlates of symptom change in schizophrenia. British Journal of Psychiatry, 194, 373-374.

Kravariti, E., Schulze, K., Kane, F., Kalidindi, S., Bramon, E., Walshe,...Murray, R.M. (2009). Stroop-test interference in bipolar disorder. British Journal of Psychiatry, 194, 285-286.

Lu, Z.L., Neuse, J., Madigan, S., \& Dosher, B.A. (2005). Fast decay of iconic memory in observers with mild cognitive impairments. Proceedings of the National Academy of Sciences of the United States of America, 102, 1797-1802.

Luo, C.R. (1999). Semantic competition as the basis of Stroop interference: evidence from color-word matching task. Psychological Science, 10, 35-40.

Machado-Pinheiro, W., Faria, A.J., Jr., Gawryszewski, L.G., \& Ribeiro-do-Valle, L.E. (2004). Experimental context modulates warning signal effects. Brazilian Journal of Medical and Biological Research, 37, 1063-1069.

MacLeod, C.M. (1991). Half a century of research on the Stroop effect: an integrative review. Psychological Bulletin, 109, 163-203.

Mitchell, R.L. (2006). Anterior cingulate activity and level of cognitive conflict: explicit comparisons. Behavioral Neuroscience, 120, 1395-1401.

Neill, W.T. (1977). Inhibitory and facilitatory processes in selective attention. Journal of Experimental Psychology: Human Perception and Performance, 3, 444-450.

Neill, W.T., \& Westberry, R.L. (1987). Selective attention and suppression of cognitive noise. Journal of Experimental Psychology: Learning, Memory and Cognition, 13, 327-334.

Niemi, P., \& Nataanen, R. (1981). Foreperiod and simple reaction time. Psychological Bulletin, 89, 133-162.

Oldfield, R.C. (1971). The assessment and analysis of handedness: the Edinburgh inventory. Neuropsychologia, 9, 97-113.

Ortells, J.J., Abad, M.J.F., Noguera, C., \& Lupianez, J. (2001). Influence of prime-probe stimulus onset asynchrony and prime precuing manipulations on semantic priming effects with words in a lexical-decision task. Journal of Experimental Psychology:
Human Perception and Performance, 27, 75-91.

Ortells, J.J., Fox, E., Noguera, C., \& Abad, M.J.F. (2003). Repetition priming effects from attended vs. ignored single words in a semantic categorization task. Acta Psychologica, 114, 185-210.

Posner, M.I., Boies, S.J., Eichelman, W.H., \& Taylor, R.L. (1969). Retention of visual and name codes of single letters. Journal of Experimental Psychology, 79, 1-16.

Roelofs, A. (2003). Goal-referenced selection of verbal action: modeling attentional control in the Stroop task. Psychological Review, 110, 88-125.

Roelofs, A. (2010). Attention, temporal predictability, and the time course of context effects in naming performance. Acta Psychologica, 133, 146-153.

Simon, J.R., \& Berbaum, K. (1988). Effect of irrelevant information on retrieval time for relevant information. Acta Psychologica, 67, 33-57.

Simon, J.R., \& Baker, K.L. (1995). Effect of irrelevant information on the time to enter and retrieve relevant information in a Stroop-type task. Journal of Experimental Psychology, 21, 1028-1043.

Stroop, J.R. (1935). Studies of interference in serial verbal reactions. Journal of Experimental Psychology, 18, 643-662.

Sugg, M.J., \& McDonald, J.E. (1994). Time course of inhibition in color-response and word-response versions of the Stroop task. Journal of Experimental Psychology: Human Perception and Performance, 20, 647-675.

Szucs, D., Soltesz, F., \& White, S. (2009). Motor conflict in Stroop tasks: direct evidence from single-trial electro-myography and electro-encephalography. Neuroimage, 47, 1960-1973.

Treisman, A., \& Fearnley, S. (1969). The Stroop test: selective attention to colours and words. Nature, 222, 437-439.

Ungar, L., Nestor, P.G., Niznikiewicz, M.A., Wible, C.G., \& Kubicki, M. (2010). Color Stroop and negative priming in schizophrenia: an fMRI study. Psychiatry Research, 181, 24-29.

Virzi, R.A., \& Egeth, H.E. (1985). Toward a translational model of Stroop interference. Memory and Cognition, 13, 304-319.

Weber, H., Biscaldi, M., \& Fischer, B. (1995). Intertrial effects of randomization on saccadic reaction times in human observers. Vision Research, 35, 2615-2642.

Zysset, S., Muller, K., Lohmann, G., \& von Cramon, D.Y. (2001). Color-word matching Stroop task: separating interference and response conflict. Neuroimage, 13, 29-36. 